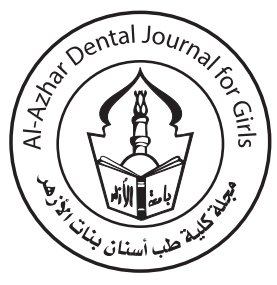

\title{
A Comparative Study Between Telescopic and Ball and Socket Attachments Used for Maxillary Implant Supported Single Dentures
}

\author{
Sherihan H. Salem ${ }^{1 *}$, Amany A. Abd el-Fattah ${ }^{2}$, Shereen M. Kabeel ${ }^{3}$, Eman M. Ibraheem ${ }^{4}$, \\ Mohamed El Zawahry ${ }^{5}$
}

Codex : 09/2001

azhardentj@azhar.edu.eg

http://adjg.journals.ekb.eg

DOI: 10.21608/adjg.2019.7707.1093

\begin{abstract}
Purpose: This study was conducted to compare between maxillary implant-supported overdentures retained by Telescopic and ball and socket regarding retention. Material and Methods: seven male patients had completely edentulous maxilla free from any systemic disease that might affect bone metabolism was selected to participate in this study. Each patient received four dental implants in the edentulous maxilla using computer guided surgical stent. Implants had enough antero-posterior spread so that load can be distributed equally. Once the implant osseointegration appears to be satisfactory, the maxillary four implants were loaded with two different attachments as follow; Group I: All patients received maxillary single denture retained with ball and socket attachments and used it for three months. Group II: After a wash up period of two weeks, all patients received another newly constructed maxillary single denture retained with telescopic attachments and used it for another three months. Retention of both groups was measured using universal testing machine. The assessment was made at time of insertion, one month and three months follow-up period. Results: Group II showed higher retention values compared to Group I. Conclusion: ball and socket attachment as well as telescopic attachment showed decrease in retention but telescopic attachment revealed a higher score than ball attachment due to frictional retention.
\end{abstract}

\section{KEYWORDS}

Implant, telescopic, ball and socket, attachment.

- Paper extracted from Doctor Thesis titled "A Comparative Study Between Telescopic and Ball and Socket Attachments Used for Maxillary Implant Supported Single Dentures"

1. Researcher Assistant of Removable Prosthodontics, Fixed and Removable Prosthodontics Department, National Research Center

2. Professor of Removable Prosthodontics and Vice Dean of Faculty of Dental Medicine for Girls, Al-Azhar University.

3. Lecturer of Removable Prosthodontics, Faculty of Dental Medicine for Girls, Al-Azhar University.

4. Associate Professor of Removable Prosthodontics, Fixed and Removable Prosthodontics Department, National Research Center.

5. Professor of Removable Prosthodontics and Chairman of Fixed and Removable Prosthodontics Department, National Research Center

* Corresponding author Email: drsherihansalem@gmail.com 


\section{INTRODUCTION}

Many patients are edentulous in one arch however the opposing arch has some or all their natural teeth, in this condition a single complete denture should be constructed. The challenge in these situations is to obtain successful complete denture for such patients who are often very difficult and sometimes it's virtually impossible ${ }^{(1)}$.

To overcome the limitations of the conventional single denture, maxillary single dentures retained by implants were successfully employed. Dental implant therapy has brought new hope for patients with maxillary edentulous arch that enhance retention, support, stability and increased biting force with the prostheses ${ }^{(2)}$.

Different attachment systems are used to improve the retention, stability and support of over dentures together with the implants, thus prolonging their durability. A widespread variety of commercially obtainable attachment systems are used to connect implants to over dentures either by splinting the implants like bars with clips or un splinting them like studs, telescopes and magnets ${ }^{(3)}$.

Studs have gained a wide popularity in clinical practice due to their simple application. Studs are solitary non-splinted attachments, less technique sensitive, and easier to clean than bars. The stud attachments consisted of a male part which is frictionally retained into the female housing that incorporated into the denture resin either directly in the mouth using self-cured or light polymerized resin, or indirectly by means of a transfer coping system and the creation of a master cast incorporating a replica of the attachment ${ }^{(4)}$.

Telescopic attachments which are also known as double crown or crown and sleeve coping (CSC) become widely used attachments. CSC consists of primary cope, permanent cementation to an abutment and a detachable secondary cope, rigidly connected to fitting surface of removable prosthesis ${ }^{(5)}$.
The telescopic attachments use has been extended to embrace implant retained prostheses to get several benefits. These retainers provide excellent retention due to frictional fit between the primary and the secondary copes, better force distribution due to axial transfer of occlusal load. Moreover, the over dentures becomes self-finding and ease for removal and insertion which inspires the patient for continuous cleaning and maintenance purposes ${ }^{(6)}$.

Concerning the use of telescopic crowns with implant-supported single dentures, only restricted data are available ${ }^{(7)}$. Hence this study was designed to estimate and compare between telescopic attachments and ball and socket attachments regarding retention.

\section{MATERIAL AND METHODS}

\section{Inclusion criteria for the selected cases}

Seven male patients having completely edentulous maxilla with well-developed ridges and healthy mucosa, aged from 50 to 60 years old, free from any systemic disease that may affect bone metabolism, nonsmokers, sufficient inter arch space not less than $11 \mathrm{~mm}$ and Angle Class I maxillomandibular relationship were enrolled in this study.

\section{Complete Maxillary Single Denture Construction}

A conventional single maxillary denture was constructed after rehabilitation and occlusal adjustment of mandibular teeth.

\section{Construction of computerized surgical stent}

The patient was asked to wear his denture after its modification by adding gutta-percha as a radio opaque detector for achieving dual scanning.

Virtual implants were placed and checked to be at least $2 \mathrm{~mm}$ away from any of the vital structures. Also $1.5 \mathrm{~mm}$ of bone was checked to be present both labially and lingually. Four virtual implants were placed in each maxillary edentulous arch and parallelism between them was checked. 
Once the positions of implants were accepted in the virtual guide, four holes were designed on the software corresponding to prefabricated metallic sleeves.

\section{Surgical Procedures}

Root formed, tapered threaded dental implants having dimensions of $3.75 \mathrm{~mm}$ diameter and the length was $10 \mathrm{~mm}$ (for canine areas) and $12 \mathrm{~mm}$ (for premolar areas) were prepared before surgery. Four implants were installed according to the flapless delayed implant placement protocol in the canine and premolar area.

\section{Prosthetic Procedures}

Once the implant osseointegration appears to be satisfactory, patients were randomly divided into two equal groups according to the attachments used for supporting the maxillary over denture as follow:

\section{A. Group I}

All patients received maxillary single denture retained with ball and socket attachments (Fig 1) and used it for three months.

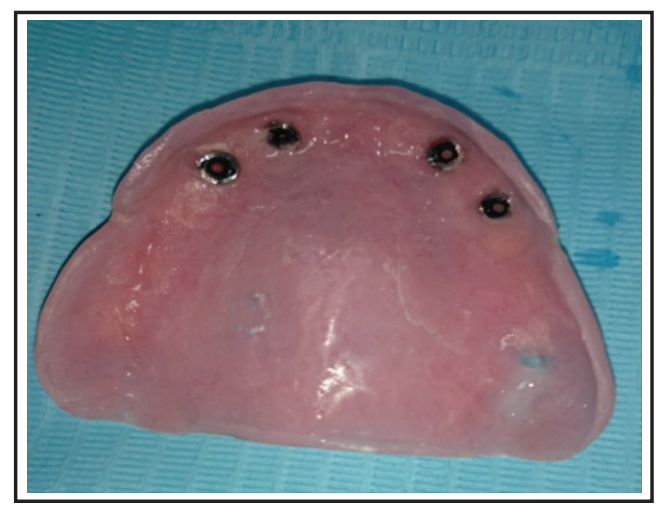

Figure(1) Denture with metallic housing and rubber rings

\section{B. Group II}

After three months, the ball and socket attachments were removed and after wash up period of two weeks the ball and socket attachments was replaced with telescopic attachments and another new denture was constructed and used for another three months.

Ball driver was used for unscrewing the ball metal attachment. Then permanent trans-mucosal titanium abutments were fastened into the implant fixtures by using octa driver till resistance achieved and then torqued up to $35 \mathrm{~N}$ using torque ratchets.

Impression copings were placed over the transmucosal abutments and then splinted for an abutment-level "open tray impression technique" (pick up type impression coping) (Fig 2). Centric jaw relation was recorded using wax wafer technique, mounting and setting up of cross linked acrylic teeth for try in steps like the conventional denture was carried out. Putty index was obtained using rubber base over labial and occlusal surface to estimate the ideal size for primary and secondary cope.

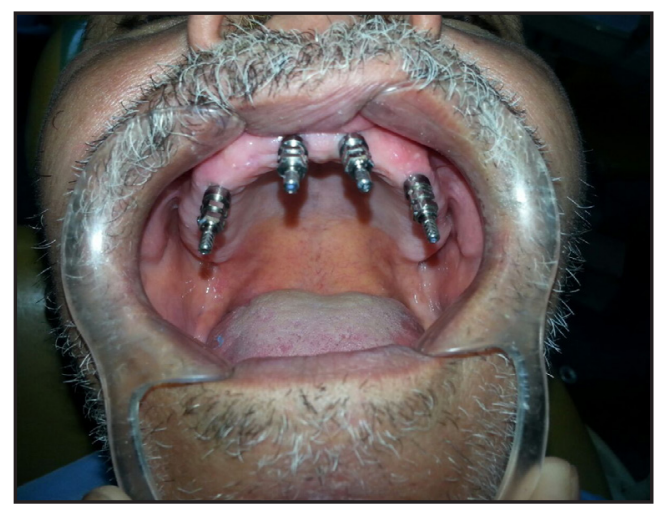

Figure (2) Impression transfer coping screwed to octa abutment using fixation long screw

Then anti-rotational plastic cap was screwed to the analogue and wax pattern of the primary coping was built up using milling wax. Final primary copings of the telescopic attachments were cast into chrome cobalt alloy.

A secondary metal coping were then fabricated over primary copings, a layer of opaque material was then used over the metal surface for esthetical demand, waxing up, try in with artificial teeth seated in proper space was then used over secondary copings and processing was then done. Final denture insertion in patient mouth was done following the same conventional steps (Fig 3). 


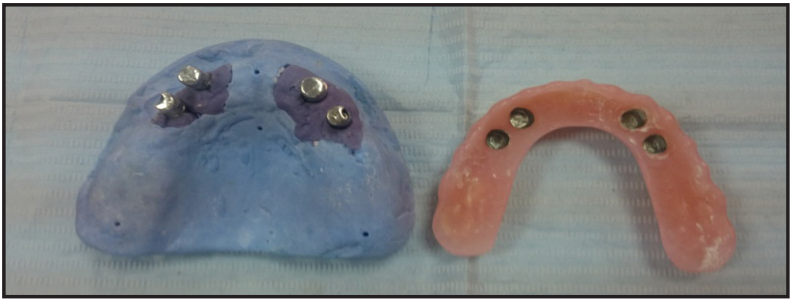

Figure (3) Primary and secondary telescope coping attached to denture.

\section{Retention Evaluation}

The universal testing machine was used to measure the retention by the aid of a metallic assembly composed of $U$-shaped metallic part resembling the denture arch curvature with two screws one at each end. The denture was modified by drill two small holes corresponding to the two screws, through which the denture in patient mouth could be attached to the metallic assembly.

The patient was asked to rest his chin on metallic chin support and to keep his face close to the metallic assembly, down ward force was then applied pass through assembly and denture till maxillary denture detached, the number recorded represent the retentive force of the denture (Fig 4).

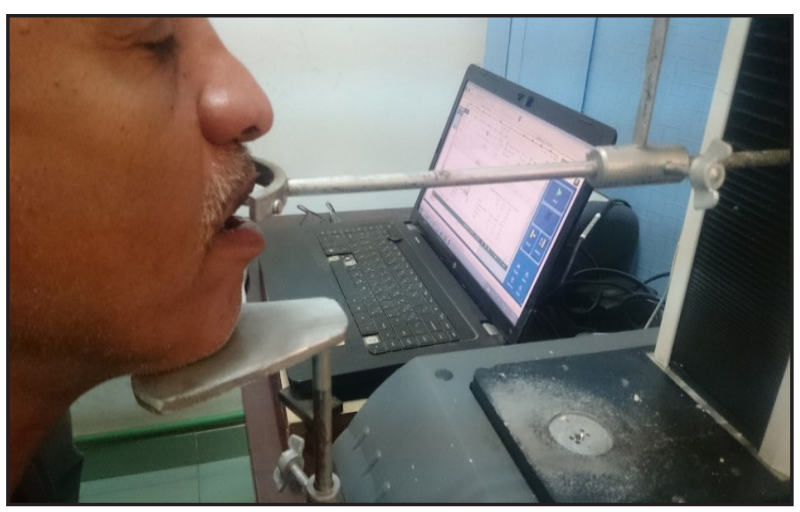

Figure (4) Patient during measurement procedure.

\section{RESULTS}

Statistical analysis was performed with SPSS 20, Graph Pad Prism and Microsoft Excel 2016. The mean values and standard deviation of the recorded values for both treatment options; Group I and Group II along the different follow up intervals (at time of insertion, after 1 month, after 3 months of follow up) were recorded and statistically analyzed.

\section{Assesment of retention:}

Evaluating effect of time on retention values through the follow up period of three months showed significant decrease in retention values as $\mathrm{P}$-value $<0.05$ (percentage of total change is 40.9 $\%$ ) for group I, while there was significant decrease in retention values as $\mathrm{P}$-value $<0.05$ (percentage of total change is $34.07 \%$ ) for group II.

Comparison between both groups: At time of insertion, group II revealed higher retention than group I with insignificant difference as P-value was 0.1568 .

While after one and three month periods group II revealed higher retention than group I with significant difference as P-value was 0.0126 and 0.0205 respectively (Table1).

Table 1: Comparison between retention of both groups at different intervals

\begin{tabular}{|c|c|c|c|}
\hline & \multicolumn{2}{|c|}{$\mathrm{M} \pm \mathrm{SD}$} & \multirow[b]{2}{*}{ P-value } \\
\hline & $\begin{array}{l}\text { Group I (First } \\
\text { denture with } \\
\text { Ball and Socket } \\
\text { Attachment) }\end{array}$ & $\begin{array}{c}\text { Group II } \\
\text { (Second } \\
\text { denture with } \\
\text { Telescopic } \\
\text { Attachment) }\end{array}$ & \\
\hline $\begin{array}{l}\text { At Time of } \\
\text { Insertion }\end{array}$ & $15.57 \pm 1.74$ & $17.14 \pm 2.13$ & 0.1568 \\
\hline One Month & $12.3 \pm 2.1$ & $15.1 \pm 1.41$ & $0.0126^{*}$ \\
\hline $\begin{array}{l}\text { Three } \\
\text { Months }\end{array}$ & $9.2 \pm 1.78$ & $11.3 \pm 1.08$ & $0.0205^{*}$ \\
\hline
\end{tabular}

\section{DISCUSSION}

All cases enrolled in this research work had completely edentulous maxilla inter-with sufficient arch distance (not less than $11 \mathrm{~mm}$ ) to provide enough space for denture base, studs and housing, copings, teeth placement, enough closest speaking space to 
fulfill the aesthetic requirements. Moreover, sufficient inter -arch space is mandatory for all cases because the retention of the telescopic attachments greatly depends on the height of abutments ${ }^{(8)}$.

Using of computerized three-dimension stent in the implant surgery have many benefits as, applying flapless surgical technique which will lead to short surgical procedure and thereby, causing less damage, less post-operative pain and swelling and less discomfort to the patient ${ }^{(9)}$.

For telescopic attachments, great degree of parallelism between the implants is required and this can be achieved by computerized stent and thereby, it enhance passive fit of superstructure, avoiding determinant lateral forces to the implants fixture and minimizing the tipping forces on the implants during insertion and removal of the prosthesis ${ }^{(10)}$.

There are no definite guidelines concerning the implants number essential to support a maxillary overdenture ${ }^{(11)}$. At least four well-spaced implants are regularly suggested for an implant-supported and retained maxillary overdenture. Increasing the number of implants used for supporting maxillary overdentures will decrease potential for single -axis fulcrum movement between attachments and will minimize the decrease in retention during functional movements ${ }^{\mathbf{( 1 2})}$. Therefore, using of more than two implants for supporting maxillary overdenture has been recommended for patients seeking removal of palatal coverage to maintain the necessary retention and comfort.

Although the use of telescopes to retain tooth supported overdentures is a well-known treatment procedure, only inadequate data are presented on the telescopic crowns use with implant supported overdentures. The results up to now designate that this treatment option can lead to expectable longstanding treatment outcomes ${ }^{(13)}$.

The main goal of this study was to compare between telescopic and ball and socket attachment with implant overdentures concerning retention.
Some studies evaluated the post-insertion prosthetic maintenance in a fully detailed 3-year followup study, in which a comparison was made between resilient telescopic crowns and ball attachments. They reported less post-insertion prosthetic care for the resilient telescopic crowns than for the ball anchors which indicate a higher retention values for telescopic crowns ${ }^{(14)}$.

The results showed decrease in retention of both telescopic overdenture with $34.07 \%$ and ball with $40.9 \%$ through the whole period of study .This results agreed with the studies performed by many authors who suggested that implant-supported telescopic attachments can be a viable alternative to the commonly used ball attachment, as application of telescopic over dentures show a long-term treatment outcome equivalent to other type of implantsupported non splinted attachments ${ }^{(10)}$.

\section{CONCLUSIONS}

- Within the limits of this study, it could be settled that Both ball and socket attachment and telescopic attachment used on implants are viable treatment options for implant supported over denture in the single edentulous maxilla

- Ball and socket attachment as well as telescopic attachment showed decrease in retention but telescopic attachment revealed a higher score than ball attachment due to frictional retention.

\section{REFERENCES}

1. Bhandari S: Outcome of single maxillary complete dentures opposing mandibular teeth: A needs to introspect on the prosthodontic treatment protocol. J Indian Prosthodont Soc. 2016; 16: 9-15.

2. Frisch E, Ziebolz D, Ratka KP, Rinke S: Double Crown Retained Maxillary Overdentures: 5 Year Follow Up. Clin Implant Dent Relat Res. 2015; 17: 22-31.

3. Kim H, Lee J, Shin S, Bryant S: Attachment systems for mandibular implant overdentures: a systematic review. J Adv Prosthodont. 2012;4:197-203. 
4. Choi JW, Yun BH, Jeong CM, Huh JB: Retentive Properties of Two Stud Attachments with Polyetherketoneketone or Nylon Insert in Mandibular Implant Overdentures. Int J Oral Maxillofac Implants. 2018;33:1079-88.

5. Dogu O, Dede M, Durmuslar C, Onur S: Telescopic Overdenture and Implant Supported Fixed Partial Denture: A Pragmatic Treatment Approach Case Report. Case Rep Dent. 2015;34:7-9.

6. Bhagat T, Walke A: Telescopic Partial Dentures-Concealed Technology. Int J Oral Health. 2015;7:143-7.

7. Rinke S, Buergers R, Ziebolz D, Roediger M: Clinical outcome of double crown-retained implant overdentures with zirconia primary crowns. J Adv Prosthodont. 2015;4: 329-37.

8. Misch C, Resnik R : Medical evaluation of the dental implant patient .3rd ed. Contemporary implant dentistry St. Louis :Mosby Elsevier .Missouri. 2008;428-40 .

9. Amarnath G, Kumar U, Hilal M, Muddugangadhar B, Anshuraj K, ShruthiC: Comparison of ConeBeam Computed Tomography, Orthopantomography with Direct Ridge Mapping for Pre-Surgical Planning to Place Implants in Cadaveric Mandibles: An Ex-Vivo Study. JIOH. 2015;7:38-42.
10. Srinivasan M, Schimmel M, Badoud I, Ammann P, Herrmann F, Müller F: Influence of implant angulation and cyclic dislodging on the retentive force of two different overdenture attachments-an in vitro study. Clin Oral Implants Res. 2015;12:23-9.

11. Kronstrom M, Widbom C, Soderfeldt B. Patient evaluation after treatment with maxillary implant-supported overdentures. Clin Implant Dent Relat Res. 2006;8:39-43.

12. Swarup S, Padmanabhan T, Kumar V: Evaluation of patient satisfaction and masticatory performance between complete dentures and single implant-supported overdentures with locator and ball attachments. J Prosth Dent. 2016;6:22-8

13. Nik SN, Nejatian T:One-Piece Implant-Retained Mandibular Overdentures By Pre-Fabricated Titanium Telescopic Attachments and Frictional Varnish: A TwoYear Prospective Study.Eur J Prosthodont Restor Dent. 2016; 24:215-21.

14. Krennmair G, lander MW, Krainhofner M, Piehslinger E: Implant supported mandibular overdentures retained with ball or telescopic crown attachments:a3 year-prospective study.Int J Prosthodont. 2006;19:164-70. 